\title{
MỌTT SỐ YẾU TỐ LIÊN QUAN ĐẾN Ý ĐỊNH SỬ DỤNG ĐỒ ĂN NHANH CỦA SINH VIÊN TRƯỜNG ĐẠI HỌC Y HÀ NỘI NĂM 2020-2021
}

\author{
Lê Thị Ngân và Phạm Bích Diệp
}

Trường Đại học Y Hà Nội

Nghiên cứu này được thực hiện với mục tiêu ứng dụng mô hình lý thuyết hành vi dụr định (TPB) để phân tích mối liên quan giữa thái độ, chuẩn mực chủ quan và nhận thức kiểm soát hành vi đến ý định sử dụng đồ ăn nhanh của sinh viên (SV) trường Đại học Y Hà Nội (ĐHYHN) năm học 2020 - 2021. Cỡ mẫu 315 SV năm 1, năm 3, năm 6 ngành Bác sĩ y học dụ̂ phòng và Cử nhân dinh dưỡng. SV có ý định sử dụng đồ ăn nhanh trong 2 tháng tới. Các yếu tố thái độ, chuẩn mực chủ quan và nhận thức kiểm soát hành vi giải thích được 10,5\% ý định sử dụng đồ ăn nhanh. Trong các yếu tố của mô hình TPB, chỉ có thái độ có ảnh hưởng đến ý định sử dụng đồ ăn nhanh $(\beta=0,32(p<0,05))$. Cần thực hiện truyền thông, sinh hoạt câu lạc bộ trong SV $Y$ để thay đổi thái độ của SV về sử dụng ăn nhanh, từ đó giúp giảm ý định sử dụng đồ ăn nhanh trong thời gian tới.

Từ khóa: đồ ăn nhanh, lý thuyết hành vi dụ định (TPB), ý định, sinh viên y

\section{I. ĐĂT VẤN ĐỀ}

Ở hầu hết các nước trên thế giới, sử dụng đồ ăn nhanh là phổ biến và chủ yếu ở người trẻ tuổi. Đối với nhiều người, đồ ăn nhanh trở thành món ăn thay thế cho những bữa cơm truyền thống vì tính tiện lợi, tiết kiệm thời gian và sự ngon miệng. Tuy nhiên, đồ ăn nhanh là loại thức ăn giàu năng lượng nhưng nghèo chất dinh dưỡng. ${ }^{1}$ Sử dụng đồ ăn nhanh thường xuyên và lâu dài sẽ ảnh hưởng đến sức khỏe, đặc biệt dẫn đến thừa cân, béo phì và có nguy cơ bị đái tháo đường type $2 .^{2-4} \mathrm{SV} Y$, là những người được trang bị kiến thức về dinh dưỡng, hiểu rõ những ảnh hưởng của đồ ăn nhanh lên sức khỏe. Tuy nhiên, SV Y cũng có lịch học lý thuyết và lâm sàng dày đặc, môi trường học tập bận rộn có thể ảnh hưởng đến thói quen ăn

Tác giả liên hệ: Phạm Bích Diệp,

Trường Đại học Y Hà Nội

Email: phambichdiep@hmu.edu.vn

Ngày nhận: 12/07/2021

Ngày được chấp nhận: 31/07/2021 uống của họ. Do đó, tìm hiểu những yếu tố liên quan đến ý định sử dụng đồ ăn nhanh là rất cần thiết để đưa ra can thiệp kịp thời.

Mô hình lý thuyết hành vi dự định (TPB) đã được áp dụng rộng rãi để phân tích các yếu tố ảnh hưởng đến ý định và hành vi. Theo TPB, ý định chịu ảnh hưởng bởi thái độ hướng đến hành vi, chuẩn mực chủ quan về hành vi và nhận thức kiểm soát hành vi. ${ }^{5}$ Một số nghiên cứu đã áp dụng mô hình TPB để giải thích ý định sử dụng đồ ăn nhanh. Nghiên cứu về hành vi sử dụng đồ ăn nhanh của nhóm học sinh và thanh niên tại Úc chỉ ra rằng các cấu phần trong mô hình TPB đã giải thích được 50\% ý định thực hiện ăn đồ ăn nhanh. ${ }^{6}$ Nghiên cứu trên nữ sinh tại Iran, thái độ, chuẩn mực chủ quan và nhận thức kiểm soát hành vi giải thích được $37,8 \%$ ý định sử dụng đồ ăn nhanh và ý định sử dụng đồ ăn nhanh giải thích được $63,8 \%$ hành vi sử dụng đồ ăn nhanh. ${ }^{7}$ Chính vì vậy, nghiên cứu này được thực hiện với mục tiêu là ứng dụng TPB để phân tích mối liên quan giữa thái 
độ, chuẩn mực chủ quan và nhận thức kiểm soát hành vi đến ý định sử dụng đồ ăn nhanh của SV trường ĐHYHN năm 2020 - 2021.

\section{II. ĐÓl TƯỢNG VÀ PHƯO'NG PHÁP}

\section{1. Đối tượng}

SV năm 1, năm 3, năm 6 ngành Bác sĩ y học dự phòng và $S V$ năm 1 , năm 3 ngành Cử nhân dinh dưỡng trường ĐHYHN.

\section{Phương pháp}

Thiết kế nghiên cứu: Nghiên cứu mô tả cắt ngang.

Thời gian nghiên cứu: Từ từ 12/2020 đến tháng 03/2021.

Địa điểm nghiên cứu: Tại Trường Đại học Y Hà Nội.

Cỡ mẫu và phương pháp chọn mẫu: Chọn mẫu toàn bộ SV (375 SV) thuộc đối tượng nghiên cứu. Có 315 SV tham gia nghiên cứu (chiếm 84\%). Cỡ mẫu 315 SV cũng đã đủ đáp ứng mục tiêu nghiên cứu vì: Cỡ mẫu tối thiểu cho phân tích nhân tố khám phá là $\mathrm{n}=5 \mathrm{x} \mathrm{m}$ ( $\mathrm{m}$ là số biến quan sát) ${ }^{8}$ do đó với $\mathrm{m}=12$ biến quan sát thì cỡ mẫu tối thiểu là 60 SV. Bên cạnh đó, cỡ mẫu tối thiểu để phân tích hồi quy tuyến tính là $n=50+8 \times$ q (q là số biến độc lập), ${ }^{9}$ do đó với $q=3$ thì cỡ mẫu tối thiểu để phân tích hồi quy là 74 SV.

Chọn mẫu nhiều giai đoạn.

Giai đoạn 1: Chọn SV Bác sĩ y học dự phòng năm 1,3 , và 6 và SV Cử nhân dinh dưỡng năm 1 và 3 .

Giai đoạn 2: Mời tất cả SV trong lớp đã chọn tham gia vào nghiên cứu và có 315 SV tham gia.

Biến số và công cụ thu thập số liệu: Bộ công cụ được xây dựng dựa trên tổng quan tài liệu về hành vi sử dụng đồ ăn nhanh và lý thuyết Hành vi dự định (TPB). ${ }^{5-7,10-13}$ Bao gồm:

Các biến số về đặc điểm nhân khẩu học: giới tính, dân tộc, tuổi, ngành học, năm học, nơi sống, người sống cùng, số lượng cửa hàng đồ ăn nhanh tại khu vực sống.

Các biến số của mô hình TPB: Các câu trả lời được thiết kế dưới dạng thang đo likert 5 mức độ (1: rất không đồng ý - 5: rất đồng ý) gồm 2 nhận định về ý định sử dụng đồ ăn nhanh, 5 nhận định về thái độ hướng tới hành vi, 4 nhận định về chuẩn mực chủ quan, 3 nhận định về nhận thức kiểm soát về hành vi.

Bộ công cụ được thiết kế dưới dạng bộ câu hỏi tự điền. Bộ câu hỏi được phát sau giờ học cho SV lớp Y1, Y3, Y6 Bác sĩ y học dự phòng và $Y 1, Y 3$ Cử nhân dinh dưỡng. SV tự điền dưới sự hướng dẫn của điều tra viên.

\section{Xử lý số liệu}

Số liệu sau khi thu thập được nhập bằng Kobotoolbox, sau đó được xử lý và phân tích bằng phần mềm Stata 15.0 .

Thống kê mô tả (tỷ lệ, giá trị trung bình, độ lệch chuẩn) được sử dụng để mô tả đặc điểm của đối tượng nghiên cứu và các cấu phần của TPB. Phân tích nhân tố khám phá (EFA) để kiểm định giá trị bộ công cụ và chỉ số Cronbach's alpha để tính toán độ tin cậy của thang đo với phương pháp rút trích nhân tố (Principal components analysis) đi cùng với phép xoay Varimax với hệ số Kaiser - Meyer - Olkin (KMO) nằm trong khoảng (giữa 0,5 và 1); kiểm định Bartlett có ý nghĩa thống kê (Sig. $<0,05)$; trị số Eigenvalue $\geq 1$ để xác định số lượng nhân tố trong phân tích EFA dựa vào hệ số tải nhân tố (Factor loading) > 0,5; tổng phương sai giải thích > 50\%. Phân tích hồi quy tuyến tính đa biến để phân tích mối liên quan với ý định sử dụng đồ ăn nhanh trong đó biến độc lập là các biến số trong mô hình TPB và biến phụ thuộc là ý định sử dụng đồ ăn nhanh. Biến độc lập và phụ thuộc được tính bằng điểm trung bình của các tiểu mục trong cùng thang đo. Giá trị $p<0,05$ là có ý nghĩa thống kê.

\section{4. Đạo đức nghiên cứu}


Nghiên cứu đảm bảo mọi nguyên tắc đạo đức trong nghiên cứu y sinh học. Sự tham gia của các SV là hoàn toàn tự nguyện, đối tượng có thể rút khỏi nghiên cứu vào bất kỳ thời điểm nào mà không có bất kỳ nguy cơ nào.

\section{KÊT QUẢ}

\section{Thông tin chung của SV tham gia nghiên cứu}

Bảng 1. Đặc điểm chung của 315 SV tham gia nghiên cứu

\begin{tabular}{|c|c|c|c|}
\hline & Đặc điểm & Số lượng (n) & Tỷ lệ (\%) \\
\hline \multirow{2}{*}{ Giới } & Nam & 80 & 25,4 \\
\hline & Nũ & 235 & 74,6 \\
\hline \multirow{2}{*}{ Dân tộc } & Kinh & 304 & 96,5 \\
\hline & Khác & 11 & 3,5 \\
\hline \multirow{3}{*}{ Năm học } & Năm 1 & 149 & 47,3 \\
\hline & Năm 3 & 91 & 28,9 \\
\hline & Năm 6 & 75 & 23,8 \\
\hline \multirow{2}{*}{ Ngành học } & Bác sĩ Y học dự phòng & 196 & 62,2 \\
\hline & Cử nhân dinh dưỡng & 119 & 37,8 \\
\hline \multirow{4}{*}{ Nơi sống } & Nhà riêng & 89 & 28,3 \\
\hline & Nhà thuê/trọ & 169 & 53,7 \\
\hline & Kí túc xá trường & 57 & 18,0 \\
\hline & Khác & 0 & 0 \\
\hline \multirow{5}{*}{ Người sống cùng } & Một mình & 25 & 7,9 \\
\hline & Bố mẹ & 69 & 21,9 \\
\hline & Anh/chị/em & 55 & 17,5 \\
\hline & Bạn bè & 164 & 52,1 \\
\hline & Khác & 2 & 0,6 \\
\hline \multirow{4}{*}{$\begin{array}{l}\text { Số lượng cửa } \\
\text { hàng ăn nhanh } \\
\text { xung quanh nơi ở }\end{array}$} & Nhiều (trên 5 cửa hàng) & 185 & 58,7 \\
\hline & Bình thường (4-5 cửa hàng) & 86 & 27,3 \\
\hline & Ít (1-3 cửa hàng) & 44 & 14,0 \\
\hline & Không có & 0 & 0 \\
\hline \multicolumn{2}{|c|}{ Tuổi (Trung bình, Độ lệch chuẩn ) } & $21,0 \pm$ & \\
\hline
\end{tabular}

Bảng 1 trình bày thông tin chung của đối tượng tham gia nghiên cứu. Độ tuổi trung bình của SV là 21,0 \pm 2,1 , chủ yếu là nữ giới $(74,6 \%)$ và dân tộc kinh $(96,5 \%)$. Trong tổng số $315 \mathrm{SV}$, chủ yếu là SV năm nhất chiếm 47,3\%; 62,2\% SV thuộc ngành Bác sĩ Y học dự phòng. Đa số SV sống tại nhà thuê/trọ $(53,7 \%)$ và sống cùng với bạn bè (52,1\%). Số lượng cửa hàng đồ ăn nhanh gần nơi ở của SV đa số là trên 5 cửa hàng $(58,7 \%)$. 


\section{Phân tích nhân tố khám phá}

\section{Bảng 2. Phân tích nhân tố khám phá}

\begin{tabular}{|c|c|c|c|}
\hline Biến & $\begin{array}{l}\text { Thái độ hưóng } \\
\text { tới hành vi }\end{array}$ & $\begin{array}{l}\text { Chuẩn mụ̣c } \\
\text { chủ quan }\end{array}$ & $\begin{array}{c}\text { Nhận thức } \\
\text { kiểm soát } \\
\text { hành vi }\end{array}$ \\
\hline Ăn đồ ăn nhanh tiết kiệm thời gian. & 0,6 & & \\
\hline Ăn đồ ăn nhanh tiện lợi. & 0,6 & & \\
\hline Tôi cảm thấy thích thú khi ăn đồ ăn nhanh. & 0,7 & & \\
\hline Đồ ăn nhanh ngon. & 0,7 & & \\
\hline $\begin{array}{l}\text { Tôi cảm thấy sảng khoái khi ăn đồ ăn } \\
\text { nhanh. }\end{array}$ & 0,8 & & \\
\hline $\begin{array}{l}\text { Khi nói đến ăn đồ ăn nhanh, tôi muốn làm } \\
\text { theo những gì bố mẹ tôi nghĩ tôi nên làm. }\end{array}$ & & 0,5 & \\
\hline $\begin{array}{l}\text { Bố mẹ tôi cho rằng ăn đồ ăn nhanh không } \\
\text { tốt cho sức khoẻ của tôi. }\end{array}$ & & 0,6 & \\
\hline $\begin{array}{l}\text { Khi nói đến ăn đồ ăn nhanh, tôi muốn làm } \\
\text { theo những gì bạn bè tôi nghĩ tôi nên làm. }\end{array}$ & & 0,8 & \\
\hline $\begin{array}{l}\text { Bạn bè tôi cho rằng ăn đồ ăn nhanh không } \\
\text { tốt cho sức khoẻ của tôi. }\end{array}$ & & 0,7 & \\
\hline $\begin{array}{l}\text { Tôi không thể chuẩn bị đồ ăn cho tôi vì bận } \\
\text { học và không có thời gian nấu nướng. }\end{array}$ & & & 0,7 \\
\hline $\begin{array}{l}\text { Tôi không thể chuẩn bị đồ ăn cho tôi vì } \\
\text { không có bếp. }\end{array}$ & & & 0,6 \\
\hline $\begin{array}{l}\text { Tôi không thể chuẩn bị đồ ăn cho tôi vì tôi } \\
\text { không muốn dành thời gian vào nấu ăn. }\end{array}$ & & & 0,6 \\
\hline Hệ số Engivalue & 2,8 & 2,0 & 2,0 \\
\hline \% Giải thích các biến số & 23,2 & 16,5 & 16,5 \\
\hline \% Lũy kế giải thích các biến số & 23,2 & 39,7 & 56,2 \\
\hline \multirow[t]{2}{*}{ Hệ số Cronbach's Alpha } & 0,8 & 0,6 & 0,7 \\
\hline & $p<0,001$ & & \\
\hline
\end{tabular}

Kết quả phân tích nhân tố khám phá cho thấy ba nhân tố rút ra giải thích được $56,2 \%$ sự biến thiên của dữ liệu và hệ số tin cậy của các thang đo của các nhân tố đều lớn hơn hoặc bằng 0,6.

\section{3. Ý định, thái độ, chuẩn mực chủ quan và nhận thức kiểm soát hành vi sử dụng đồ ăn nhanh}

SV có ý định sử dụng đồ ăn nhanh trong 2 tháng tới ở mức phân vân (trung bình = 3,2). Thái độ hướng đến sử dụng đồ ăn nhanh và chuẩn mực chủ quan về sử dụng đồ ăn nhanh là dao động ở mức phân vân và đồng ý (trung bình $=3,5$ ), trong khi đó nhận thức kiểm soát hành vi ở mức không đồng ý và phân vân (trung bình = 2,6). 
Bảng 3. Điểm trung bình về ý định, thái độ, chuẩn mực chủ quan và nhận thức kiểm soát hành vi sử dụng đồ ăn nhanh của SV

\section{Biến số}

Mức độ đồng ý

Trung bình Độ lệch chuẩn

\begin{tabular}{|c|c|c|}
\hline Ý định sử dụng đồ ăn nhanh trong 2 tháng tới. & 3,2 & 1,0 \\
\hline Cố gắng ăn đồ ăn nhanh ít nhất 1 lần trong 2 tháng tới. & 3,2 & 1,0 \\
\hline Trung bình thang đo ý định & 3,2 & 0,7 \\
\hline Ăn đồ ăn nhanh tiết kiệm thời gian. & 3,7 & 0,8 \\
\hline Ăn đồ ăn nhanh tiện lợi. & 3,7 & 0,8 \\
\hline Tôi cảm thấy thích thú khi ăn đồ ăn nhanh. & 3,4 & 0,9 \\
\hline Đồ ăn nhanh ngon. & 3,6 & 0,8 \\
\hline Tôi cảm thấy sảng khoái khi ăn đồ ăn nhanh. & 3,2 & 0,9 \\
\hline Trung bình thang đo thái độ & 3,5 & 0,6 \\
\hline $\begin{array}{l}\text { Khi nói đến ăn đồ ăn nhanh, tôi muốn làm theo những gì bố } \\
\text { mẹ tôi nghĩ tôi nên làm. }\end{array}$ & 3,0 & 1,0 \\
\hline $\begin{array}{l}\text { Bố mẹ tôi cho rằng ăn đồ ăn nhanh không tốt cho sức khoẻ } \\
\text { của tôi. }\end{array}$ & 4,1 & 0,9 \\
\hline $\begin{array}{l}\text { Khi nói đến ăn đồ ăn nhanh, tôi muốn làm theo những gì bạn } \\
\text { bè tôi nghĩ tôi nên làm. }\end{array}$ & 3,3 & 1,0 \\
\hline $\begin{array}{l}\text { Ban bè tôi cho rằng ăn đồ ăn nhanh không tốt cho sức khoẻ } \\
\text { của tôi. }\end{array}$ & 3,5 & 0,9 \\
\hline Trung bình thang đo chuẩn mực chủ quan & 3,5 & 0,7 \\
\hline $\begin{array}{l}\text { Tôi không thể chuẩn bị đồ ăn cho tôi vì tôi bận học và không } \\
\text { có thời gian nấu nướng. }\end{array}$ & 2,9 & 1,0 \\
\hline Tôi không thể chuẩn bị đồ ăn cho tôi vì tôi không có bếp. & 2,5 & 1,2 \\
\hline $\begin{array}{l}\text { Tôi không thể chuẩn bị đồ ăn cho tôi vì tôi không muốn dành } \\
\text { thời gian vào nâu ăn. }\end{array}$ & 2,5 & 1,1 \\
\hline Trung bình thang đo nhận thức kiểm soát hành vi & 2,6 & 0,9 \\
\hline
\end{tabular}

Ghi chú: 1 "rất không đồng ý", 2 "không đồng ý", 3 "phân vân", 4 "đồng ý", 5 "rất đồng ý".

\section{Mối liên quan đến ý định sử dụng đồ ăn nhanh của SV trong 2 tháng tới}

Kết quả phân tích hồi quy đa biến cho thấy R2 hiệu chỉnh $=0,105(p<0,05)$ có nghĩa các biến số của mô hình TPB giải thích được 10,5\% ý định sử dụng đồ ăn nhanh trong 2 tháng tới. Thái độ hướng đến hành vi có mối liên quan thuận chiều đến ý định sử dụng đồ ăn nhanh trong 2 tháng tới với $\beta=0,32(p<0,05)$. 


\section{Bảng 4. Mối liên quan đến ý định sử dụng đồ ăn nhanh trong 2 tháng tới của SV}

\begin{tabular}{lccc}
\hline \multicolumn{1}{c}{ Biến số } & Hệ số chuẩn hóa $(\boldsymbol{\beta})$ & $\mathbf{P}$ & VIF \\
\hline Thái độ & 0,32 & 0,00 & 1,06 \\
\hline Chuẩn mực chủ quan & $-0,01$ & 0,84 & 1,06 \\
\hline Nhận thức kiểm soát hành vi & 0,05 & 0,28 & 1,01 \\
\hline $\mathbf{R}^{2}$ & & 0,105 & \\
\hline
\end{tabular}

\section{BÀN LUÂNN}

Nghiên cứu phân tích một số yếu tố liên quan đến ý định sử dụng đồ ăn nhanh của SV trường ĐHYHN dựa trên mô hình TPB. Nghiên cứu cho thấy SV có thái độ tương đối tích cực đối với sử dụng đồ ăn nhanh: trung bình = 3,5 so với điểm tối đa là 5 (tức là khoảng $70 \%$ ). Kết quả này cao hơn nghiên cứu trong SV Hàn Quốc cho thấy điểm thái độ trong sử dụng đồ ăn nhanh là 24,55 trên tổng điểm 48 (tức khoảng $51 \%) .{ }^{13,14}$ Các tiểu mục có thái tích cực là đồ ăn nhanh tiện lợi, tiết kiệm thời gian và ngon. Đây cũng là kết quả phù hợp với các nghiên cứu khác như học sinh Hàn Quốc có thái độ đồng ý cao ở tiểu mục hợp khẩu vị, ${ }^{13}$ hay hương vị ngon. ${ }^{15}$

Nghiên cứu này cho thấy ý định của SV về sử dụng đồ ăn nhanh trong 2 tháng tới là phân vân: trung bình ý định là 3,2 trong khi đó điểm tối đa là 5 (64\%). Bên cạnh đó nhận thức kiểm soát hành vi có điểm trung bình thấp (trung bình = 2,6). Phần lớn SV không đồng ý với các nhận định là SV không thể nấu nướng hay chuẩn bị đồ ăn. Kết quả mô hình hồi quy tuyến tính đa biến cho thấy "thái độ hướng tới hành vi" có liên quan thuận chiều với ý định sử dụng đồ ăn nhanh trong 2 tháng tới với $\beta=0,32(p<0,05)$, có nghĩa là SV càng có thái độ tích cực đối với đồ ăn nhanh, thì ý định sử dụng đồ ăn nhanh càng cao. Kết quả này tương tự nghiên cứu trên SV tại Úc và các nghiên cứu tại Iran. 6,7,10,12 Tuy nhiên, kết quả từ học sinh của Hàn Quốc lại cho thấy thái độ không có mối liên quan đến ý định sử dụng đồ ăn nhanh. ${ }^{13}$ Như vậy, mối liên quan đến ý định sử dụng đồ ăn nhanh theo mô hình TPB là khác nhau tuỳ thuộc vào đối tượng và địa điểm nghiên cứu. Đối với $S V$ trường ĐHYHN, thái độ hướng đến sử dụng đồ ăn nhanh là yếu tố ảnh hưởng mạnh nhất. Sự khác biệt này có thể do SV $Y$ có quỹ thời gian hạn chế, áp lực từ việc học tập, thực hành lâm sàng, thi cử diễn ra thường xuyên và liên tục nên sự tiện lợi và tiết kiệm thời gian được SV ưu tiên hơn cả. Mặc dù kết quả nghiên cứu này chưa cho thấy mối liên quan giữa chuẩn mực chủ quan với ý định thực hiện hành vi. Tuy nhiên, điểm trung bình về chuẩn mực chủ quan cũng tương tự như thái độ hướng đến hành vi (trung bình $=3,5$ ) trong đó nhận thức về quan điểm của bố mẹ và bạn bè cho rằng đồ ăn nhanh có hại cho sức khoẻ có điểm trung bình cao nhất (tương ứng là 4,1 và 3,5). SV Y là SV của một khối ngành đặc biệt, họ có kiến thức về y học và được đào tạo về dinh dưỡng, nên ý định thực hiện hành vi của họ chịu ảnh hưởng lớn từ thái độ của chính họ.

Mô hình phân tích hồi quy tuyến tính đa biến cho thấy các thành phần của TPB đã giải thích 10,5\% ý định sử dụng đồ ăn nhanh của SV trường ĐHYHN $(p<0,05)$. Kết quả này thấp hơn nhiều so với nghiên cứu khác ví dụ như TPB giải thích được 37,8\% ý định sử dụng đồ ăn nhanh trong nghiên cứu của của Nooshin Rouhani-Tonekaboni trên nhóm đối tượng nữ sinh tại Iran, ${ }^{7}$ hay $50 \%$ trong nghiên cứu tại Úc. ${ }^{6}$ Kết quả nghiên cứu gợi ý rằng ý định sử dụng 
đồ ăn nhanh của SV trường ĐHYHN có thể chịu ảnh hưởng lớn từ các yếu tố bên ngoài.

Nghiên cứu có hạn chế như cỡ mẫu mới chỉ thực hiện trong phạm vi sinh viên hệ cử nhân dinh dưỡng và bác sĩ y học dự phòng của Trường Đại học $Y$ Hà Nội. Đây là nhóm SV được đào tạo nhiều kiến thức về dinh dưỡng hơn các SV chuyên ngành khác trong trường, do vậy kết quả nghiên cứu này chưa thể ngoại suy cho tất cả SV trong trường. Mô hình TPB mới chỉ đề cập các yếu tố liên quan ở cấp độ cá nhân, cần nghiên cứu thêm các yếu tố khác ngoài cá nhân có ảnh hưởng đến ý định sử dụng đồ ăn nhanh của $S V$ để cung cấp thêm bằng chứng cho can thiệp.

\section{KÉT LUÂN}

Ý định sử dụng đồ ăn nhanh của SV trường ĐHYHN ở mức vừa phải. Thái độ về sử dụng đồ ăn nhanh của SV ở mức tích cực, đặc biệt là thái độ về tính tiện lợi, ngon và không mất thời gian. Tuy nhiên, thái độ không đồng ý với các nhận định là không thể nấu nướng/chuẩn bị đồ ăn do bận học của SV là cao. Các yếu tố của mô hình TPB giải thích 10,5\% ý định sử dụng đồ ăn nhanh của SV trường ĐHYHN. Thái độ hướng tới hành vi sử dụng đồ ăn nhanh có mối liên quan tương đối chặt chẽ đến ý định sử dụng đồ ăn nhanh của SV. Cần có những nghiên cứu tiếp theo về các yếu tố bên ngoài ảnh hưởng đến ý định sử dụng đồ ăn nhanh của SV để cung cấp thêm bằng chứng đầy đủ về các yếu tố ảnh hưởng. Bên cạnh đó, nhà trường cần có những buổi truyền thông giúp SV thay đổi thái độ với sử dụng đồ ăn nhanh và lập kế hoạch quản lý tốt thời gian trong các hoạt động học tập và cuộc sống. Ngoài ra, câu lạc bộ SV nên tổ chức các buổi chia sẻ kinh nghiệm về kỹ năng nấu ăn, tiết kiệm thời gian và đảm bảo dinh dưỡng.

\section{TÀI LIẸU THAM KHẢO}

1. Role of food prepared away from home in the American diet, 1977-78 versus 199496: changes and consequences - PubMed. Accessed October 25, 2020. https://pubmed. ncbi.nlm.nih.gov/12047838/

2. Stender S, Dyerberg J, Astrup A. Fast food: unfriendly and unhealthy. Int $J$ Obes (Lond). 2007; 31(6): 887-890. doi:10.1038/ sj.ijo.0803616

3. Global, regional and national prevalence of overweight and obesity in children and adults 1980-2013: A systematic analysis. Accessed December 16, 2020. https://www.ncbi.n/m.nih. gov/pmc/articles/PMC4624264/

4. Jeffery RW, Baxter J, McGuire M, Linde J. Correction: Are fast food restaurants an environmental risk factor for obesity? Int J Behav Nutr Phys Act. 2006; 3: 35. doi:10.1186/14795868-3-35

5. Ajzen I. The theory of planned behavior. In: Organizational Behavior and Human Decision Processes. 1991:179-211.

6. Dunn KI, Mohr P, Wilson CJ, Wittert GA. Determinants of fast-food consumption. An application of the Theory of Planned Behaviour. Appetite. 2011; 57(2): 349-357. doi:10.1016/j. appet.2011.06.004

7. Rouhani-Tonekaboni N, Seyedi-Andi SJ, Haghi M. Factors Affecting Fast Food Consumption Behaviors of Female Students in North of Iran: Application of Theory of Planned Behavior. Caspian Journal of Health Research. 2018; 3(3): 75-79. doi:10.29252/cjhr.3.3.75

8. Wilson Van Voorhis CR, Morgan BL. Understanding Power and Rules of Thumb for Determining Sample Sizes. TQMP. 2007; 3(2): 43-50. doi:10.20982/tqmp.03.2.p043

9. Green SB. How Many Subjects Does It Take To Do A Regression Analysis. Multivariate 
Behav Res. 1991; 26(3): 499-510. doi:10.1207/ s15327906mbr2603_7

10. Sharifirad G, Yarmohammadi $P$, Azadbakht L, Morowatisharifabad MA, Hassanzadeh A. Determinants of Fast Food Consumption among Iranian High School Students Based on Planned Behavior Theory. J Obes. 2013. doi:10.1155/2013/147589

11. Mazloomy-Mahmoodabad $\mathrm{S}$, Mahbobirad M, Asadpour M, VaeziA, Fallahzadeh $H$, Mahmoodabadi $H$. Determiners of fast-food consumption in Iranian university students: Application of prototype/willingness model. Journal of Education and Health Promotion. 2020; 9: 345. doi:10.4103/jehp.jehp_466_20

12. Akbay C, Tiryaki GY, Gul A. Consumer characteristics influencing fast food consumption in Turkey. Food Control. 2007; 18(8): 904-913. doi:10.1016/j.foodcont.2006.05.007

13. Seo H, Lee S-K, Nam S. Factors influencing fast food consumption behaviors of middle-school students in Seoul: an application of theory of planned behaviors. Nutr Res Pract. 2011; 5(2): 169. doi:10.4162/nrp.2011.5.2.169

14. Choi MK. A Study on the Relationship between Fast Food Consumption Patterns and Nutrition Knowledge, Dietary Attitude of Middle and High School Students in Busan. Culinary science and hospitality research. 2007; 13(2): 188-200.

15. Han MJ. A Survey of College Student Behaviors on Fast Food Restaurants in Seoul Area. Journal of the Korean Society of Food Culture. 1992; 7(2): 91-96.

\section{Summary}

\section{ASSOCIATED FACTORS WITH INTENTION TO USE FAST FOOD OF STUDENTS AT HANOI MEDICAL UNIVERSITY, ACADEMIC YEAR 2020-2021}

This study was conducted with the aim of applying the theory of planned behavior (TPB) to analyze the relationship between attitude, subjective norm and perceived behavioral control and intention to use fast food among students at Hanoi Medical University in the academic year 2020 - 2021. Sample size was 315 students among the first, third and sixth year Doctor of preventive medicine and Bachelor of Nutrition. Students intend to use fast food in the next 2 months. Attitude forward behavior, subjective norm and perceived behavioral control explained $10.5 \%$ of intention to use fast food. Only attitude forward behavior has an effect on intention to use fast food $(\beta=0.32$ ( $p$ $<0.05)$ ). It is necessary to provide health education among medical students to change students' attitude about using fast food, thereby helping to reduce the intention to use fast food in the future.

Keywords: fast food, the theory of planned behavior (TPB), intention, medical students. 